\title{
COVID-19 and the return to head and neck outpatient activity in the United Kingdom: what is the new normal?
}

\author{
Sumrit Bola ${ }^{1} \cdot$ Dominic Jaikaransingh ${ }^{1} \cdot$ Stuart C Winter ${ }^{1,2}$
}

Received: 8 September 2020 / Accepted: 13 October 2020 / Published online: 6 November 2020

(c) The Author(s) 2020

\begin{abstract}
Purpose As surgical specialties now begin the graduated return to elective activity and face-to-face clinics, this paper investigates the current head and neck outpatient practices across the United Kingdom.

Methods A cross-sectional study comprised of an online 20-item survey was distributed to members of the British Association of Head \& Neck Oncologists (BAHNO). The survey was open on a web-based platform and covered topics including safety measures for patients, protective equipment for healthcare staff and protocols for the use of flexible nasendoscopy in the clinic.

Results The survey was completed by 117 participants covering 66 NHS Trusts across the UK. There was a significant reduction in face-to-face Otolaryngology, Maxillofacial and Speech and Language clinic patients when compared to prepandemic numbers $(p<0.0001)$. Risk assessments for flexible nasendoscopy were done for $69 \%$ of clinics and $58 \%$ had an established protocol. Room downtime after flexible nasendoscopy ranged from 0 to $6 \mathrm{~h}$ and there was a significant increase in allocated downtime after a patient had coughed/sneezed $(p<0.001)$. Natural ventilation existed in $36 \%$ of clinics and the majority of responders didn't know the Air Change Per Hour (ACPH) of the clinic room (77\%). Where ACPH was known, it often did not match the allocated room downtime.

Conclusion There is a wide variation in outpatient activity across the United Kingdom, but adaptations are being made to try and maintain staff and patient safety. However, more can still be done by liaising with allied teams to clarify outpatient protocols.
\end{abstract}

Keywords Outpatient clinic $\cdot$ Otolaryngology $\cdot$ SALT $\cdot$ Air change per hour · Aerosol-generating procedure

\section{Introduction}

The COVID-19 pandemic caused by the SARS-CoV-2 coronavirus infection has resulted in an unprecedented challenge on healthcare systems worldwide [1,2]. The reduction in clinical activity and elective surgical work has been due to a number of factors including access to primary care, risk of

Electronic supplementary material The online version of this article (https://doi.org/10.1007/s00405-020-06458-x) contains supplementary material, which is available to authorized users.

Stuart C Winter

stuart.winter@nds.ox.ac.uk

1 Otolaryngology Department, Oxford University NHS Foundation Trust, Headley Way, Oxford OX3 9DU, UK

2 Nuffield Department of Surgical Sciences, University of Oxford, Oxford, UK nosocomial infection and the redeployment of staff $[3,4]$. As outpatient services adopt new strategies for the delivery of care, many face-to-face appointments have been cancelled, causing a significant backlog of surgical work.

Early evidence suggested that Head and Neck clinicians, for example, those in specialities such as Otolaryngology and Maxillofacial surgery, were at a particularly high risk of contracting the disease [5]. This was attributed to the elevated viral load found in the nasopharynx [6], close proximity to the oral cavity and the frequency of aerosol-generating procedures [7]. Outpatient management of head and neck cancer extends beyond these two specialities with a broad range of multidisciplinary team members performing at risk procedures such as nasendoscopy, speech valve changes and tracheostomy care.

With no established cure or vaccine for COVID-19 to date, the virus is likely to be a burden for the foreseeable future, and there has been extensive published guidance on 
working during COVID-19 and the return to elective activity $[8,9]$. As the first peak of the pandemic passed across most of Europe [10], surgical departments began a graduated return to selective face-to-face clinics.

\section{Objective}

The aim of this paper was to investigate the current head and neck outpatient practices across the United Kingdom as faceto-face clinics are reinstated. We explore safety measures for patients, protective equipment for healthcare staff and protocols around the use of flexible nasendoscopy (FNE) in the clinic. In particular, we investigate if COVID-19 related safety advice [11] is being implemented appropriately.

\section{Materials and methods}

This cross-sectional study comprised of an online 20-item survey which was distributed via email to members of the British Association of Head \& Neck Oncologists (BAHNO). Members include the multi-disciplinary healthcare professionals involved in the study and treatment of head and neck cancer and related conditions. The email invitation was directed at those members who performed flexible nasendoscopy (purposive sampling). Prior to distribution, a pre-test of the survey was sent to senior committee members for question composition feedback. Questions were then modified and transferred to a web-based platform (SurveyMonkey, San Mateo, CA, U.S.A. https://www.surveymonkey.com) where it was completed by a small pilot group $(n=5)$ and these results were not included in the main analysis. The study design was based on established survey reporting guidance [12].

The survey was open from July 1st to July 21 st 2020 and email invitations were sent twice during this period. Questions were asked with regards to current and former outpatient practices (Online Appendix A). Topics included:

1 Personal protective equipment used in clinic (PPE)

2 Number of patients booked into a clinic

3 Patient screening prior to the clinic appointment

4 Risk assessment and protocols for flexible nasendoscopy

5 Outpatient room ventilation and room air changes per hour (ACPH)

6 Downtime associated with an Aerosol-Generating Procedure (AGP).

For the purpose of this study, flexible nasendoscopy was not classified as AGP unless the patient had coughed or sneezed during the examination. Downtime of a room was defined as the time when a room was left to 'rest' prior to cleaning. In this time period, air particles are allowed to fall to the ground or be replaced by the ventilation system and no staff or patient should re-enter the room.

To prevent the entry of duplicate data from the same hospital, participants were asked to name their place of work. Realising that some NHS Trust may have remote sites for outpatient clinics, entries were only excluded from the study if the place of work and data entry were identical. A paired samples t-test was used to determine if there was a statistically significant difference in the downtime after an AGP and the number of patients booked into a clinic. Data were analysed as means and there was a comparison between current activity and that prior to the pandemic.

\section{Results}

\section{Demographics}

The survey was sent to 420 members of BAHNO and was completed by 117 (28\%) participants. The results captured details of outpatient activity across 66 different NHS Trusts in England, Wales, Scotland and Ireland (Fig. 1). After assessing to see if entries were duplicated, 11 participants were eliminated from the study leaving 106 completed questionnaires for analysis. There was a broad range of responders reflecting the different specialisms who perform nasendoscopy; Otolaryngologists $(58 \%, n=62)$, Speech and Language Therapists $(22 \%, n=24)$, Maxillofacial surgeons $(16 \%, n=17)$ and Oncologists $(3 \%, n=3)$. It was not known what proportion of the overall membership performed flexible nasendoscopy. The majority of responders were from Tertiary Centres $(60 \%, n=64)$.

\section{Patients per clinic}

Before the COVID-19 pandemic, the number of patients that otolaryngologists had booked into a 4-h outpatient clinic ranged from 6 to 15 for a single clinic and 14-20 for a joint head and neck cancer (HNC) follow-up clinic, with the majority of clinicians seeing 12 patients $($ mean $=12.8$, standard deviation $=2.5$ ). The post-pandemic figures were between 2 and 14 patients in a 4-h clinic for both single and HNC clinics (mean $=6.5, \mathrm{SD}=2.3$ ), with otolaryngology responders reporting a 50\% mean reduction in outpatient appointments $(p<0.0001, t=17.28)$. On average, a new appointment was 1.9 times longer (minutes allocated) than appointments prior to the pandemic. Three otolaryngology responders reported no reduction in booked outpatient clinic numbers; two of these were in district general hospitals and one from a tertiary centre.

Pre-pandemic maxillofacial clinics ranged from 8 to 14 booked patients for a single $4-\mathrm{h}$ clinic and $18-40$ patients for 
Fig. 1 Survey responders across England, Ireland, Scotland and Wales

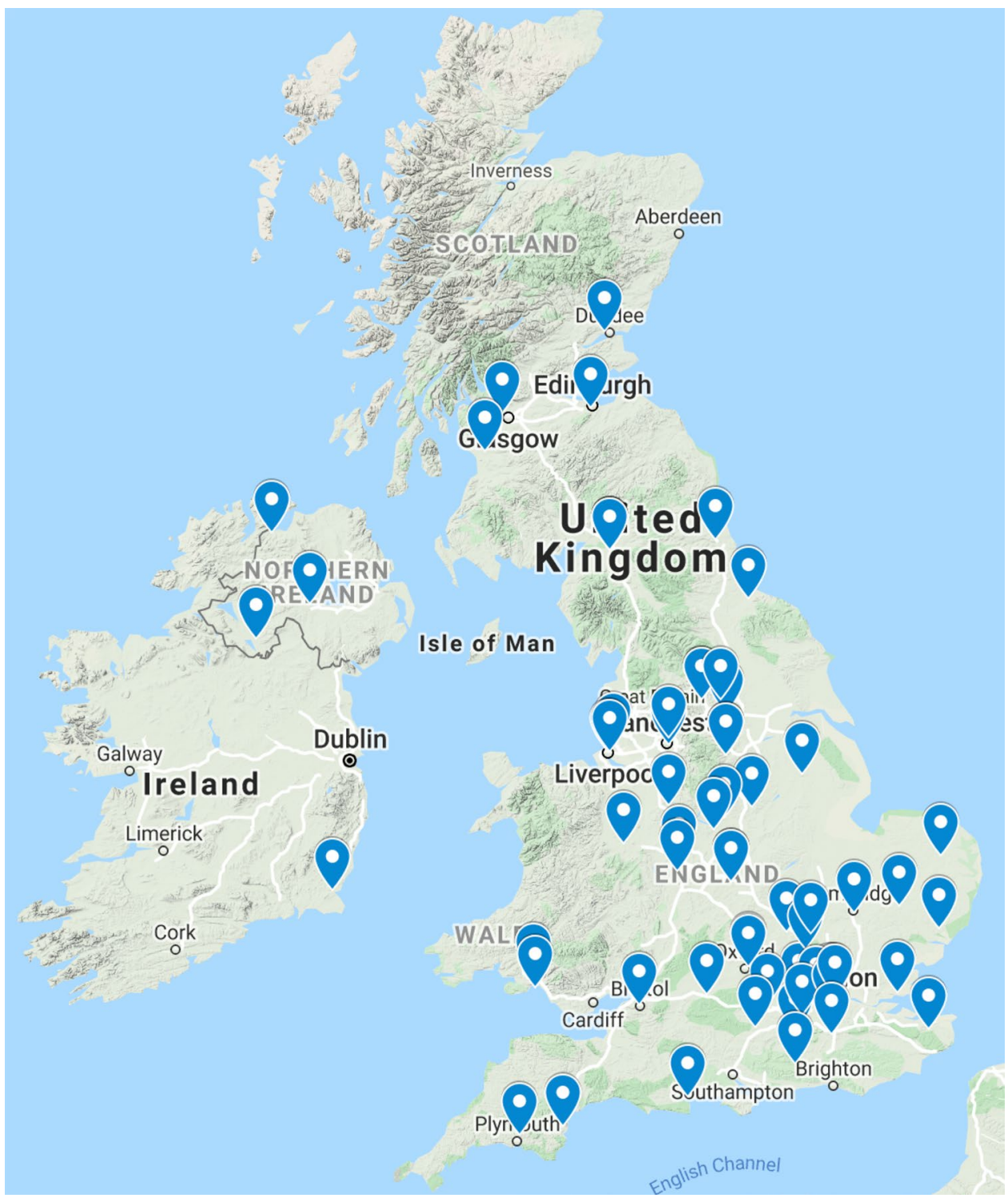

a shared $\mathrm{HNC}$ follow-up joint clinic ( mean $=18, \mathrm{SD}=7.7)$. Current maxillofacial clinics range from 4 to 10 booked patients for a single clinic and 10-16 for a joint $\mathrm{HNC}$ follow up clinic (mean =7.4, SD=3.0). Maxillofacial responders reported a significant reduction in patient numbers ( $p<0.0001, t=5.99)$ as opposed to the oncology responders who reported that 12-18 patients were booked pre-pandemic versus 6-12 patients post-pandemic; there was no significant difference in post-pandemic activity for oncology.

Speech and Language Therapist reported to having 4-8 patients booked into a specialist clinic (for example voice or dysphagia) and 8-20 into a joint HNC clinic prior to the pandemic (mean $=9.1, \mathrm{SD}=5.5$ ). Post-pandemic numbers were $1-4$ and 6-16 patients respectively (mean $=3.9, \mathrm{SD}=3.0$ ). Although one responder reported no change in their numbers for joint HNC clinic, there was an overall significant reduction in booked patients post-pandemic $(p<0.0001, t=6.04)$.

\section{Patient screening prior to outpatient appointment}

Patient screening prior to the outpatient appointment was done with temperature checks, letter advice for self-vetting and symptoms screening by the nurse or receptionist (Table 1). Eighty-seven percent of responders knew the screening methods used and most departments (56\%) used two or more screening methods prior to clinical review. One responder reported that COVID-19 swabs were being performed prior to a speech and language clinic for Fibreoptic Endoscopic Evaluation of Swallow (FEES) but swabs were not carried out for the otolaryngology appointments in the same NHS Trust. In two otolaryngology departments, patient symptom screening was done by the clinician prior to entering the clinic room and this was the only method used.

In the majority of clinics, patients were reported to 'always' wear a mask during the consultation $(62 \%)$ or 
Table 1 Screening tools utilised by Otolaryngology, Maxillofacial, Oncology and SALT Outpatient clinics

\begin{tabular}{ll}
\hline Screening tool & $\begin{array}{l}\text { Number of clinics where } \\
\text { screen is implemented }\end{array}$ \\
\hline Symptoms screening by nurse or receptionist & 58 \\
Patient letter advising not to attend if symptomatic & 54 \\
Temperature taken prior to consultation & 42 \\
Telephone screening prior to appointment & 15 \\
Symptoms screening by a clinician at the consultation & 6 \\
COVID swab prior to appointment & 2 \\
Hospital entrance questionnaire & 1 \\
Don't know if/how patients are screened & 2 \\
\hline
\end{tabular}

Multiple screening tools implemented for each clinic 'sometimes' during the consultation (12\%). In $25 \%$ of departments, mask wearing for patients was not standard practice. Four departments reported the utilisation of a mask with a hole for nasendoscopy. There was no significant difference in the reported frequency of patient mask wearing between Otolaryngology, SALT, Oncology and Maxillofacial responders.

\section{Flexible nasendoscopy and personal protective equipment}

All otolaryngology survey responders were still performing flexible nasendoscopy (FNE) in outpatient clinic whilst 5 non-otolaryngologist reported that FNE was no longer performed due to safety concerns or an unsuitable room. Seventy-three respondents (69\%) reported having had a risk assessment for performing FNE in outpatients and of these, $61(58 \%)$ had a protocol for the procedure. Thirtythree responders (31\%) reported not having a protocol or not knowing if there was a protocol for performing FNE in an outpatient clinic. Overall, the majority of survey responders found that nasendoscopy took 10 min longer than normal to account for donning/doffing and not having an assistant. This did not include the room cleaning time or room downtime in between patients.

For those still performing FNE in clinic, 94 responders (93\%) reported wearing Level 2 personal protective equipment (PPE), two respondents reported wearing Level 3 PPE and the remaining 5 reported that they wore Level 1 PPE. The scope was most often performed in a designated flexible nasendoscopy room that was shared between clinicians $(42 \%)$ or in a nasendoscopy room allocated to each clinician $(30 \%)$. When asked how the nasendoscopy room was prepared, $84 \%$ of responders had a video screen available and $25 \%$ had acquired disposable FNE scopes (Table 2).
Table 2 How the allocated room for flexible nasendoscopy is prepared

\begin{tabular}{ll}
\hline & $\begin{array}{l}\text { Number of } \\
\text { respondents }\end{array}$ \\
\hline Cleared of unnecessary items & 92 \\
Video screen availability & 85 \\
Single endoscopist in the room & 63 \\
Availability of disposable scopes & 25 \\
Surfaces cleaned after the procedure (Clinic staff) & 92 \\
Deep clean after the procedure (Cleaners) & 3 \\
\hline
\end{tabular}

Multiple preparations for each room

\section{Room ventilation}

When asked about the ventilation of the room where flexible nasendoscopy was performed, many responders $(n=36)$ reported natural ventilation only via open windows. Eighteen departments reported that their rooms had no ventilation and 15 responders were unsure what ventilation existed in their nasendoscopy room. Four clinics were described as having negative pressure rooms and 20 responders described having a positive pressure air-conditioned room (Fig. 2).

\section{Downtime associated with aerosol-generating procedure}

Survey participants were asked about room downtime when performing flexible nasendoscopy and if there was any difference in downtime if a patient had coughed or sneezed as this was classified as an aerosol-generating event (Fig. 3). From the comments, we found that some rooms were being left as long as $6 \mathrm{~h}$ after nasendoscopy. Most departments had a different protocol if an aerosol-generating event had taken place in that the room downtime was longer when the patients had coughed/sneezed compared to when an aerosolgenerating event had not taken place $(p<0.001)$. 
Fig. 2 Room ventilation of the allocated flexible nasendoscopy room in the clinic (NP Negative pressure)

\section{What ventilation exists in the room where nasendoscopy is performed?}

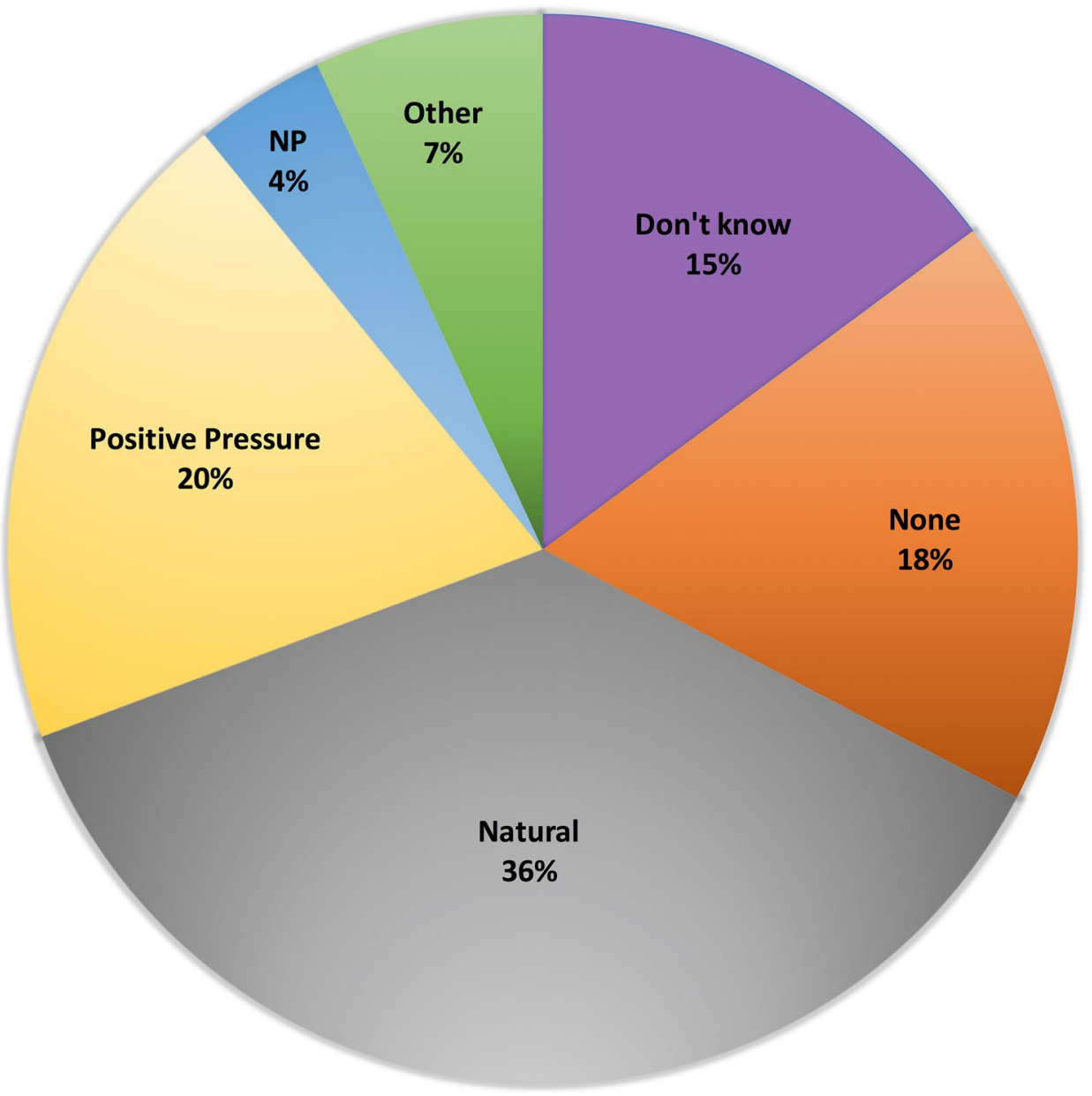

Twenty-five responders knew the number of air changes per hour $(\mathrm{ACPH})$ of their nasendoscopy room which ranged from 0 to 30 between the clinics (Table 3). In 14 responses, the ACPH did not clear $99 \%$ of room air particles in the reported allocated downtime after an AGP [11]. The majority of responders (75\%) were unaware of the ACPH of their nasendoscopy room, however, $92 \%$ had a policy for room downtime after an AGP.

\section{Discussion}

These results show a variety of outpatient head and neck clinical activity across the United Kingdom but there were some unifying themes across the different departments. Apart from the Oncologist, all responders reported a significant reduction in outpatient numbers and an increase in the time allocated to each new outpatient appointment. In the comments section, this was attributed to waiting room capacity, availability of audiology, reduced nursing staff and the need to allocate rooms for flexible nasendoscopy. The survey responses did not account for the number of clinics that could run simultaneously, so the estimated reduction in patient numbers is not likely to represent the full extent of the ongoing backlog. It is, therefore, important that strategies are developed to address limited space in outpatient clinics [13].

As social distancing rules continue to be modified in the United Kingdom, it is essential to screen patients for symptoms prior to clinical review. The majority of responders reported at least two COVID-19 screening methods prior to the clinic appointment. Recognising that the virus has a highly infectious but asymptomatic prodrome period, many departments had protocols in place for patients to wear facemasks. There is evidence to support mouth covering reduces droplet production [14] and since the survey closed, it has become compulsory for the public to wear masks whilst indoors [15].

There have been a number of recommendations as to how flexible nasendoscopy (FNE) should be performed safely. Although it is not an aerosol-generating procedure, it involves the manipulation of high viral load nasopharyngeal 


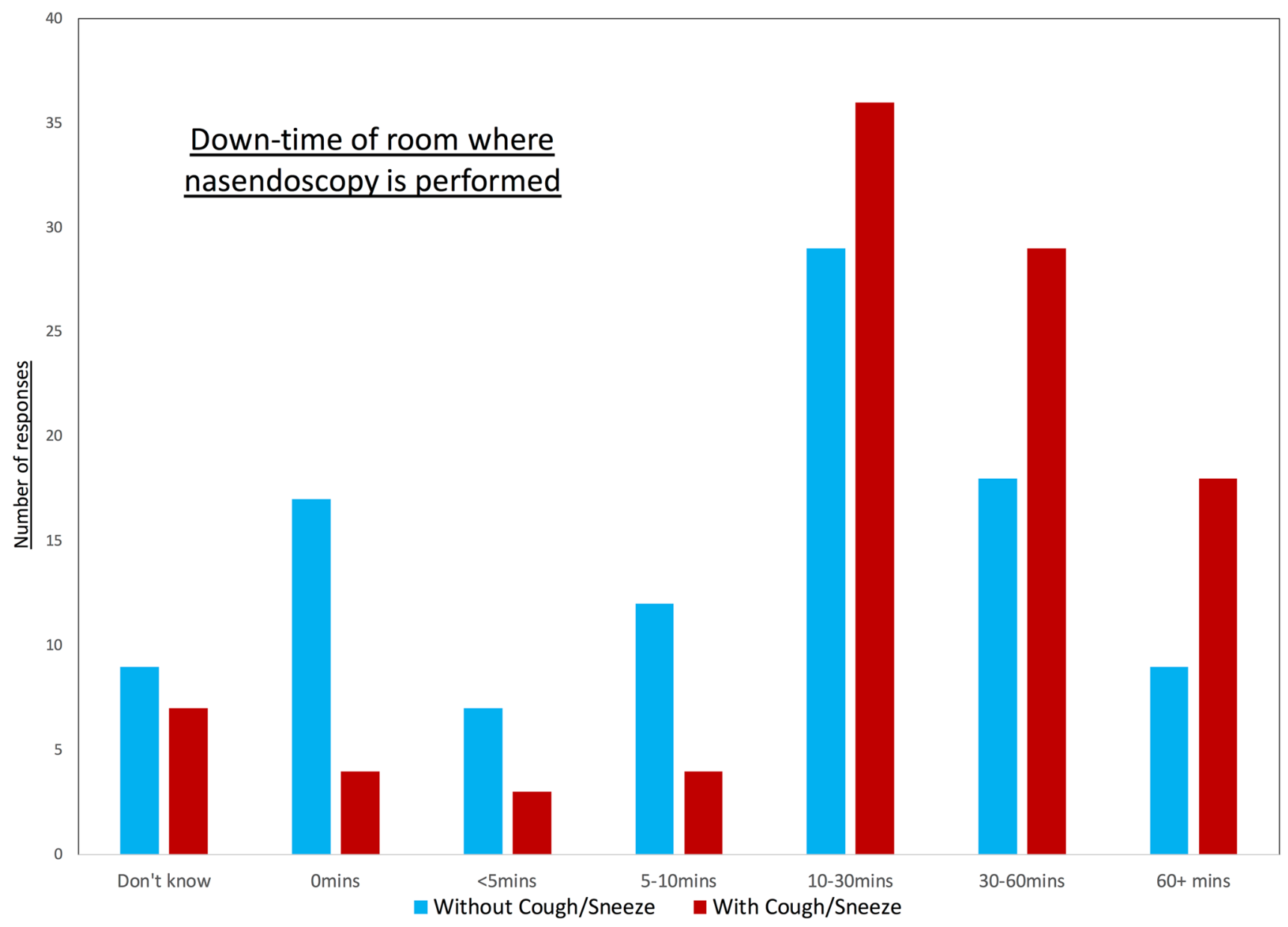

Fig. 3 Downtime allocated to flexible nasendoscopy room after an aerosol-generating procedure has taken place. (Downtime $=$ minutes where room is left untouched after patient and clinician have left)

tissue and may cause an asymptomatic infected patient to cough or sneeze. This results in the aerosolisation of virus particles from the respiratory tract which become airborne and contaminate multiple surfaces in the environment [16]. There were reports of inadequate FNE risk assessments, however, the majority of responders wore Level 2 personal protective equipment for flexible nasendoscopy in the outpatient setting which is in keeping with ENT-UK guidance, Royal College of Speech and Language Therapists guidance and recommendations from outside the United Kingdom [11, 17-19]. Although one SALT department was performing COVID-19 swabs prior to clinic review, there is no guidance recommending this and there is evidence to suggest screening at a time of low virus prevalence can be harmful [20].

In terms of room ventilation and downtime, the protocols for FNE have to be interpreted in the context of each individual hospital; taking into account the physical space and ventilation in which the procedure is taking place. The survival time of SARS-CoV-2 varies considerably by surface type and in aerosol form, viable particles can remain after $3 \mathrm{~h}$ [21]. In settings where rooms are re-used after an AGP, this poses a risk not only to clinical staff but also to subsequent patients entering the room. Although there was less downtime allocated to when a patient had not coughed or sneezed, the allocated downtime and ACPH sometimes did not match. For example, where an ACPH was reported to be 6 , we would expect 46 min downtime after an AGP as this removes $99 \%$ of the air particles in that room making it safe for the next patient [11]. We instead saw a range of times from 5 min to $6 \mathrm{~h}$ after an AGP (patient coughing or sneezing). It was not clear how the downtime was calculated or why any downtime was allocated when a patient had not coughed or sneezed. It was also concerning that even though some rooms had no ventilation, potential AGPs were still taking place. 
Table 3 Room downtime and Air Changes Per Hour (ACPH) reported by responders

\begin{tabular}{|c|c|c|c|}
\hline $\begin{array}{l}\text { Specialty and number of } \\
\text { ACPH of clinic room }\end{array}$ & $\begin{array}{l}\text { Reported downtime after } \\
\text { AGP (minutes) }\end{array}$ & $\begin{array}{l}\text { Does this clear } 99 \% \text { of } \\
\text { room air particles? }\end{array}$ & $\begin{array}{l}\text { Does this clear } \\
90 \% \text { of room air } \\
\text { particles? }\end{array}$ \\
\hline Otolaryn 0 & $10-30$ & No & No \\
\hline Otolaryn 0 & $10-30$ & No & No \\
\hline Otolaryn 3 & $60+$ & Yes & Yes \\
\hline Otolaryn 4 & $60+$ & Yes & Yes \\
\hline OMFS 4 & $30-60$ & No & Yes \\
\hline OMFS 5 & $30-60$ & Yes & Yes \\
\hline Otolaryn 6 & $10-30$ & No & Yes \\
\hline Otolaryn 6 & $30-60$ & Yes & Yes \\
\hline Otolaryn 6 & $60+$ & Yes & Yes \\
\hline SALT 6 & $60+$ & Yes & Yes \\
\hline SALT 6 & $10-30$ & No & Yes \\
\hline OMFS 6 & $10-30$ & No & Yes \\
\hline OMFS 6 & $30-60$ & Yes & Yes \\
\hline OMFS 6 & $5-10$ & No & No \\
\hline OMFS 6 & $30-60$ & Yes & Yes \\
\hline Otolaryn 8 & $10-30$ & No & Yes \\
\hline Otolaryn 11 & $30-60$ & Yes & Yes \\
\hline Otolaryn 12 & $10-30$ & Yes & Yes \\
\hline Otolaryn 12 & $10-30$ & Yes & Yes \\
\hline Otolaryn 17 & 0 & No & No \\
\hline Otolaryn 18 & $10-30$ & Yes & Yes \\
\hline Otolaryn 20 & $5-10$ & No & Yes \\
\hline OMFS 22 & $10-30$ & Yes & Yes \\
\hline OMFS 25 & $5-10$ & No & Yes \\
\hline SALT 30 & $10-30$ & Yes & Yes \\
\hline
\end{tabular}

$A G P$ aerosol-generating procedure, Otolaryn Otolaryngology, SALT Speech and Language Therapy, OMFS Maxillofacial Surgery

\section{Limitations}

Electronic surveys are known to have a small response rate. However, the survey email invitation was aimed at those members of BAHNO who performed flexible nasendoscopy and so the actual response rate is likely to be higher than reported. This survey is also limited to head and neck clinics which have taken priority over other elective work and so the figures may not represent the full extent of the surgical backlog amongst the specialties.

\section{Conclusion}

The continuing presence of COVID-19 in the population will prevent the immediate return to pre-pandemic clinical activity [22]. Adaptations are being made across the UK to try and maintain staff and patient safety, but more can still be done by liaising with hospital infectious diseases and the hospital estates team to clarify outpatient protocols.
From this survey, we consider the current standard practice to be:

- Level 2 PPE and video screen availability for nasendoscopy

- Reduced number of patients per face-to-face clinic

- Minimum of two patient-screening methods prior to clinician review

- Patient wears masks during clinical consultation

- Longer downtime for nasendoscopy room when patient has coughed/sneezed.

From this survey, we have found that.

- Many clinicians do not know air changes per hour of their nasendoscopy room

- Most outpatient departments have natural ventilation only

- Level 1 PPE for flexible nasendoscopy is not common

- There will be a considerable backlog of outpatient work. 
Author contributions SB: Methodology, data analysis and original draft preparation. DJ: Data processing and manuscript editing. SW: Conceptualisation and review.

\section{Compliance with ethical standards}

Conflict of interest The authors have no relevant financial or non-financial interests to disclose.

Open Access This article is licensed under a Creative Commons Attribution 4.0 International License, which permits use, sharing, adaptation, distribution and reproduction in any medium or format, as long as you give appropriate credit to the original author(s) and the source, provide a link to the Creative Commons licence, and indicate if changes were made. The images or other third party material in this article are included in the article's Creative Commons licence, unless indicated otherwise in a credit line to the material. If material is not included in the article's Creative Commons licence and your intended use is not permitted by statutory regulation or exceeds the permitted use, you will need to obtain permission directly from the copyright holder. To view a copy of this licence, visit http://creativecommons.org/licenses/by/4.0/.

\section{References}

1. Wiersinga W, Rhodes A, Cheng A et al (2020) Pathophysiology, transmission, diagnosis, and treatment of coronavirus disease 2019 (COVID-19). JAMA. https://doi.org/10.1001/ jama.2020.12839

2. Anderson R, Heesterbeek H, Klinkenberg D, Hollingsworth T (2020) How will country-based mitigation measures influence the course of the COVID-19 epidemic? The Lancet 395(10228):931934. https://doi.org/10.1016/s0140-6736(20)30567-5

3. Mahase E (2020) Covid-19: urgent cancer referrals fall by $60 \%$, showing "brutal" impact of pandemic. BMJ. https://doi. org/10.1136/bmj.m2386

4. Collaborative $\mathrm{C}$ (2020) Global guidance for surgical care during the COVID-19 pandemic. Br J Surg 107(9):1097-1103. https:// doi.org/10.1002/bjs. 11646

5. Lu D, Wang H, Yu R, Yang H, Zhao Y (2020) Integrated infection control strategy to minimize nosocomial infection of coronavirus disease 2019 among ENT healthcare workers. J Hosp Infect 104(4):454-455. https://doi.org/10.1016/j.jhin.2020.02.018

6. Zou L, Ruan F, Huang M et al (2020) SARS-CoV-2 viral load in upper respiratory specimens of infected patients. N Engl J Med 382(12):1177-1179. https://doi.org/10.1056/nejmc2001737

7. Workman AD, Jafari A, Welling DB et al (2020) Airborne aerosol generation during endonasal procedures in the era of COVID19: risks and recommendations. Otolaryngol Head Neck Surg 163(3):465-470. https://doi.org/10.1177/0194599820931805

8. Couloigner V, Schmerber S, Nicollas R et al (2020) COVID-19 and ENT Surgery. Eur Ann Otorhinolaryngol Head Neck Dis 137(3):161-166. https://doi.org/10.1016/j.anorl.2020.04.012

9. Mehanna H, Hardman J, Shenson J et al (2020) Recommendations for head and neck surgical oncology practice in a setting of acute severe resource constraint during the COVID-19 pandemic: an international consensus. Lancet Oncol 21(7):E350-E359
10. Dong E, Du H, Gardner L (2020) An interactive web-based dashboard to track COVID-19 in real time. Lancet Infect Dis 20(5):533-534. https://doi.org/10.1016/s1473-3099(20)30120-1

11. A graduated return to elective ENT within the COVID-19 pandemic. Accessed 4, Aug 2020 from Entuk.org. https://www.entuk .org/graduated-return-elective-ent-within-covid-19-pandemic-0. Published 2020.

12. Burns KEA, Duffett M, Kho ME et al (2008) A guide for the design and conduct of self-administered surveys of clinicians. Can Med Assoc J 179(3):245-252. https://doi.org/10.1503/cmaj.08037 2

13. Paleri V, Hardman J, Tikka T, Bradley P, Pracy P, Kerawala C (2020) Rapid implementation of an evidence-based remote triaging system for assessment of suspected referrals and patients with head and neck cancer on follow-up after treatment during the COVID-19 pandemic: Model for international collaboration. Head Neck 42:1674-1680

14. Anfinrud P, Stadnytskyi V, Bax C, Bax A (2020) Visualizing speech-generated oral fluid droplets with laser light scattering. N Engl J Med 382(21):2061-2063. https://doi.org/10.1056/nejmc 2007800

15. Face coverings: when to wear one and how to make your own. GOV.UK. Accessed 31, July 2020 from https://www.gov.uk/gover nment/publications/face-coverings-when-to-wear-one-and-howto-make-your-own/face-coverings-when-to-wear-one-and-howto-make-your-own. Published 2020.

16. Ong SWX, Tan YK, Chia PY et al (2020) Air, surface environmental, and personal protective equipment contamination by severe acute respiratory syndrome coronavirus 2 (SARS-CoV-2) from a symptomatic patient. JAMA 323(16):1610. https://doi. org/10.1001/jama.2020.3227

17. Royal College of Speech and Language Therapists, 2020d, Speech and language therapist-led endoscopic procedures in the COVID-19 pandemic. Accessed 22 Aug 2020 form www.rcslt.org. 2020.

18. Chan J, Wong E, Lam W (2020) Practical aspects of otolaryngologic clinical services during the 2019 novel coronavirus epidemic. JAMA Otolaryngol Head Neck Surg 146(6):519

19. Vukkadala N, Rosenthal E, Patel Z (2020) In response to COVID19 and the otolaryngologist: preliminary evidence-based review. Laryngoscope. https://doi.org/10.1002/lary.28672

20. Watson J, Whiting P, Brush J (2020) Interpreting the COVID-19 test result. BMJ. https://doi.org/10.1097/PHM.000000000000147 1

21. Van Doremalen N, Bushmaker T, Morris D et al (2020) Aerosol and surface stability of SARS-CoV-2 as compared with SARSCoV-1. New Engl J 382:1564-1567

22. Wang D, Hu B, Hu C, Zhu F, Liu X, Zhang J et al (2020) Clinical characteristics of 138 hospitalized patients with 2019 novel coronavirus-infected pneumonia in Wuhan, China. JAMA 323(11):1061

Publisher's Note Springer Nature remains neutral with regard to jurisdictional claims in published maps and institutional affiliations. 